\title{
GROWTH OF COMPOSITE ENTIRE FUNCTIONS
}

\author{
By ZHEN-ZHONG ZHOU
}

\section{Introduction.}

Singh had proved the following result in his paper [2]. Let $f(z)$ and $g(z)$ be entire functions of finite order satisfying $\rho_{g}<\lambda_{f} \leqq \rho_{f}$ and $g(0)=0$. Here $\rho$ and $\lambda$ indicate the order and the lower order of indexed entire function, respectively. Then

$$
\limsup _{r \rightarrow \infty} \frac{\log T(r, f(g))}{T(r, f)} \leqq \rho_{f} .
$$

In this paper we shall prove the following

THEOREM. under the same assumptions as in Singh's result

$$
\lim _{r \rightarrow \infty} \frac{\log T(r, f(g))}{T(r, f)}=0 .
$$

This Theorem shows that corollary 1 of Singh's paper [2] does not have any meaning at all. In fact Singh assumed

$$
\liminf _{r \rightarrow \infty} \frac{\log T(r, f(g))}{T(r, f)} \geqq \rho_{f} .
$$

and $\rho_{g}<\lambda_{f} \leqq \rho_{f}$. Hence $\rho_{f}$ should be positive, But Theorem shows that

$$
\lim _{r \rightarrow \infty} \frac{\log T(r, f(g))}{T(r, f)}=0 .
$$

This is a contradiction. This shows that

$$
\liminf _{r \rightarrow \infty} \frac{\log T(r, f(g))}{T(r, f)} \geqq \rho_{f}>0
$$

does not occur.

\section{Proof of Theorem.}

Firstly it is possible to choose $\varepsilon>0$ so small that

Received February 27, 1986

$$
\rho_{g}+2 \varepsilon<\lambda_{f}-\varepsilon,
$$


since $\rho_{g}<\lambda_{f}$. Then there exists $r_{0}(\varepsilon)$ so that

$$
r^{\lambda_{f-(\varepsilon / 2)}}<T(r, f)<r^{\rho_{f}+\varepsilon}, \quad \log M(r, g)<r^{\rho_{g}+\varepsilon}
$$

for all $r>r_{0}(\varepsilon)$ Further it is possible to assume that $\rho_{f}+\varepsilon<r^{\varepsilon}$. Niino and Suita [1] showed that

$$
\log T(r, f(g(z))) \leqq \log T(M(r, g), f)
$$

Hence for $r>r_{0}(\varepsilon)$

$$
\begin{aligned}
\log T(r, f(g(z))) & <\left(\rho_{f}+\varepsilon\right) \log M(r, g) \\
& <\left(\rho_{f}+\varepsilon\right) r^{\rho_{g}+\varepsilon} \\
& <r^{\rho+2 \varepsilon} \\
& <r^{\lambda_{f}-\varepsilon} .
\end{aligned}
$$

Hence

$$
\frac{\log T(r, f(g(z)))}{T(r, f)}<\frac{r^{\lambda} f^{-\varepsilon}}{r^{\lambda} f^{-(\varepsilon / 2)}}=r^{-(\varepsilon / 2)} .
$$

This gives the desired result:

$$
\lim _{r \rightarrow \infty} \frac{\log T(r, f(g(z)))}{T(r, f)}=0
$$

\section{REFERENCES}

[1] K. Ninno And N. Suita, Growth of a composite function of entire functions, Kodai Math. J., 3 (1980), 374-379.

[2] A.P. SingH, Growth of composite entire functions, Kodai Math. J., 8 (1985), 99-102.

Department of MATHematics,

ANHUi Normal University,

Wuhu, Anhui, China 